\title{
A Preliminary Study on the Relationship Between High-resolution Computed Tomography and Lung Function in People at Risk of Developing Chronic Obstructive Pulmonary Disease
}

\section{Rui Lv}

The First Affiliated Hospital of Wenzhou Medical University

\section{Huaqian Jin}

The First Affiliated Hospital of Wenzhou Medical University

\section{Mengyao Xie}

The First Affiliated Hospital of Wenzhou Medical University

\section{Pingping Shu}

The First Affiliated Hospital of Wenzhou Medical University

\section{Mingli Ouyang}

The First Affiliated Hospital of Wenzhou Medical University

\section{Yanmao Wang}

The First Affiliated Hospital of Wenzhou Medical University

\section{Dan Yao}

The First Affiliated Hospital of Wenzhou Medical University

\section{Lehe Yang ( $\nabla$ yanglehe@wmu.edu.cn )}

The First Affiliated Hospital of Wenzhou Medical University

\section{Xiaoying Huang}

The First Affiliated Hospital of Wenzhou Medical University

\section{Yiran Wang}

The First Affiliated Hospital of Wenzhou Medical University

\section{Research}

Keywords: COPD, HRCT, pulmonary function, the air trapping index, mean expiratory lung density

Posted Date: October 12th, 2021

DOl: https://doi.org/10.21203/rs.3.rs-955212/v1

License: (c) (i) This work is licensed under a Creative Commons Attribution 4.0 International License. Read Full License 
Page $2 / 21$ 


\section{Abstract}

Background: There is high morbidity and mortality for patients with chronic obstructive pulmonary disease (COPD) in China. The aim of our study was to explore the differences in high-resolution computed tomography (HRCT) emphysema parameters, air trapping parameters, and lung density parameters between patients at high risk and low risk of developing COPD and evaluate their correlation with lung function indicators.

Methods: In this retrospective, single-center cohort study, we enrolled outpatients from the Physical Examination Center and Respiratory Medicine of The First Affiliated Hospital of Wenzhou Medical University. The patients at risk of developing COPD were $\geq 40$ years-old, had chronic cough or sputum production, and/or had exposure to risk factors for the disease and had not reached the diagnostic criteria. Patients were divided into a low-risk group and high-risk group according to $\mathrm{FEV} 1 / \mathrm{FVC} \geq 80 \%$ and $80 \%>F E V 1 / F V C \geq 70 \%$. The data on clinical characteristics, clinical symptom score, lung function, and HRCT were recorded.

Results: 72 COPD high-risk patients and 86 COPD low-risk patients were enrolled in the study, and the air trapping index of the left, right, and bilateral lungs of the high-risk group were significantly higher than those of the low-risk group. Additionally, the mean lung density during expiration was significantly lower than that of the low-risk group. The emphysema index of left, right, and bilateral lungs was negatively correlated with FEV1/FVC (correlation coefficients were -0.33-0.22-0.26). There was a negative correlation between the air trapping index of left and right lungs and bilateral lungs and FEV1/FVC(correlation coefficients were $-0.33-0.23-0.28$, respectively), and the mean expiratory lung density of left and right lungs and bilateral lungs was positively correlated with FEV1/FVC (correlation coefficients were 0.31 , $0.25,0.29$, respectively).

Conclusions: The air trapping index and the mean expiratory lung density obtained by HRCT combined with post-processing technology can be used as a basis for distinguishing between people at high risk and low risk for developing COPD. Emphysema index, air trapping index, and mean expiratory lung density shows significantly negative correlation with FEV1/FVC, and this can be used to assess the pulmonary function status of people at risk of developing COPD.

\section{Background}

Chronic obstructive pulmonary disease (COPD) is a common preventable and treatable disease that is characterized by persistent airflow limitation that is usually progressive and not completely reversible [1]. The late stage of the disease is often accompanied by systemic multi-system chronic diseases, including cardiovascular diseases, metabolic syndrome, osteoporosis, depression, anxiety, and lung cancer, which contribute to the overall severity in patients $[2,3]$.

The clinical diagnosis of COPD is primarily based on pulmonary function tests (PFTs) [4], but some constraints remain in detecting early changes in lung structure or function. As a general function test, the 
accurate assessment of local pulmonary function damage is difficult. Also, it has been reported in the literature that clinical symptoms will be present or changes in pulmonary function tests (PFTs) will appear when more than $30 \%$ of the total lung parenchyma has been destroyed [5]. Although the forced expiratory volume in one second percentage (FEV1\%) may be unchanged, there can be significant changes in imaging findings [6]. The Global Strategy for the Diagnosis, Management, and Prevention of Chronic Obstructive Pulmonary Disease 2020 report suggests that further in-depth research is needed to study those patients without evidence of airflow limitation but who have evidence of structural lung disease upon chest imaging, such as emphysema, that is consistent with what is found in patients with COPD [7].

Computed tomography (CT) is the modality of choice for the imaging characterization of COPD patients, and it can be used to describe the changes in lung parenchyma in patients with COPD [8]. In the past years, with the rapid development of high-resolution computed tomography (HRCT) technology and continuous development of image post-processing and reconstruction techniques, CT imaging has been recognized as an important method for the assessment of COPD [9]. The emphysema index (EI) [10, 11], air trapping index (ATI) [12-14], and mean lung density (MLD) $[15,16]$ are the most common functional imaging parameters. However, these have rarely been evaluated in a population of patients at risk for developing COPD.

The primary aim of our research was therefore to evaluate the differences in HRCT emphysema parameters, air trapping parameters, and lung density parameters between patients with high and low risk of developing COPD, and to investigate the relationships between CT metrics and lung function indicators.

\section{Methods}

This retrospective and single-center cohort study was carried out at The First Affiliated Hospital of Wenzhou Medical University in China. Ethical approval was obtained, and all participants consented to be included in the trial.

\section{Recruitment and enrolment}

There were 158 participants aged 40-79 years with no prior diagnosis of COPD who were recruited from the Physical Examination Center, Department and Respiratory Medicine and other departments between March 2018 and May 2019 (Figure 1). They underwent respiratory biphasic HRCT, pulmonary function tests, and filled out three detailed questionnaires. The risk of developing COPD was determined by the forced expiratory volume in the first second of expiration to forced vital capacity (FEV1/FVC): low risk (FEV1/FVC $\geq 80 \%)$, or high risk ( $80 \%>\mathrm{FEV} 1 / \mathrm{FVC} \geq 70 \%)$.

Exclusion criteria were as follows: asthma, severe pneumonia, tuberculosis, pulmonary fibrosis, or other concomitant chronic diseases, including cardiovascular disease, skeletal muscle dysfunction, metabolic syndrome, osteoporosis, depression, anxiety, and lung cancer. 
Medical information and patient characteristics including age, sex, height $(\mathrm{m})$, weight $(\mathrm{kg})$, smoking index, and BMI were obtained from screening questionnaires. All data were stored in an encrypted database.

\section{Clinical Scores and Questionnaires}

All subjects were asked to independently complete three questionnaires, the modified Medical Research Council (mMRC), the chronic obstructive pulmonary disease Assessment Test (CAT), and the clinical chronic obstructive pulmonary disease questionnaire (CCQ). After exhaustive examination, the researchers then recorded the data from the completed questionnaires.

\section{Spirometry}

Spirometry was performed using a Vostro15 portable spirometer according to the ATS/ERS standard. The percentage predicted values were calculated using the equations for Asian adults supplied in the user's manual. After a 10-min rest, the subject assumed a seated position with feet in full contact with the floor, breathed for three cycles, then forcefully, rapidly, and deeply inhaled through the interface device and suddenly, continuously, and steadily exhaled to reach a maximum amount of breathing. It was necessary for the exhalation process to not be spontaneously interrupted by unsolicited coughing. Measurements were performed in triplicate for each subject, and each curve was coincident, as far as possible, to qualify the PFT, which included FVC, FEV1, FEV1\%prep, and FEV1/FVC.

\section{Chest HRCT examination and image analysis}

Prior to HRCT scanning, patients underwent breathing training to optimize the measurement of maximum inspiration. HRCT was performed at suspended full inspiration and expiration using a Gem Energy Spectroscopy CT (Discovery HD750, GE, USA). Scanning parameters were as follows: tube voltage 120 $\mathrm{kV}$, automatic tube current (mA automatic modulation technique), pitch 0.984 , slice thickness $5 \mathrm{~mm}$ with $1.25 \mathrm{~mm}$ reconstruction interval, detector coverage $40 \mathrm{~mm}$, X-ray tube rotation speed 0.6 slice/rotation, DFOV $30 \mathrm{~cm}$, SFOV $50 \mathrm{~cm}$, and a $512 \times 512$ matrix.

Quantitative assessments of emphysema were performed using Volume Viewer 11.3 software. We established limits, and the computer program calculated the attenuation as the mean lung density (MLD) of the whole lung. Then, we calculated the total lung area, and the lung area occupied by attenuation values lower than previously fixed thresholds (-950 HUs at inspiration and $-856 \mathrm{HUs}$ at expiration) by Lung VCAR.

\section{Statistical analysis}

Statistical analyses were performed using IBM SPSS (Statistical Package for the Social Sciences) Statistics Software (version 22.0, IBM). The measurement data were tested for normality by the Kolmogorov-Smirnov method. Continuous variables of normal distribution are presented as the mean \pm standard deviation and were compared by two-sample t-test. Continuous variables of skewed distribution 
are expressed as median values (interquartile ranges) and were compared by the Mann-Whitney U-test. Categorical variables are expressed as a number (\%) and were compared by the $\chi^{2}$ test or Fisher's exact test. The correlation between HRCT parameters and pulmonary function parameters was assessed by the Spearman rank correlation test. $p<0.05$ was considered statistically significant.

\section{Results}

\section{Baseline Characteristics}

The mean age of 158 subjects was $52.18 \pm 8.56$ years, ranging from 40 to 76 years (Table 1 ). There were 123 males and 35 females. Overall, 86 (54.4\%) participants were under a low risk of developing COPD and 72 were (45.5\%) in the high-risk group. There was no statistical difference in BMI, smoking index, or exposure to dust between the two groups. Compared with subjects in the high-risk group, those in the lowrisk group had similar scores for mMRC, CAT, and CCQ ( $p=0.53, p=0.47$, and $p=0.84$, respectively). The FEV1/FVC and FEV1\%prep in thte low-risk group were significantly lower than those in the high-risk group $(0.82 \pm 0.07$ vs. $0.79 \pm 0.08,0.92 \pm 0.14$ vs. $0.86 \pm 0.13, p<0.05)$, while no difference was found in FEV1 and $\mathrm{FVC}(\mathrm{p}=0.47$ and $p=0.93$, respectively $)$. 
Table 1

Demographics and baseline characteristics of the study subjects

\begin{tabular}{|llll|}
\hline $\begin{array}{l}\text { Demographics and baseline } \\
\text { characteristics }\end{array}$ & $\begin{array}{l}\text { Low-risk group } \\
(\mathbf{n}=86)\end{array}$ & $\begin{array}{l}\text { High-risk group } \\
(\mathbf{n}=72)\end{array}$ & $\begin{array}{l}\text { P } \\
\text { value }\end{array}$ \\
\hline Age (years) & $51.17 \pm 7.30$ & $52.73 \pm 8.39$ & 0.06 \\
\hline Sex & - & - & 0.98 \\
\hline Male & $67(77.9 \%)$ & $56(77.8 \%)$ & - \\
\hline Female & $19(22.1 \%)$ & $16(22.2 \%)$ & - \\
\hline Height (m) & $(1.62,1.71)$ & $(1.60,1.71)$ & 0.18 \\
\hline Weight (kg) & $65.05 \pm 9.07$ & $66.89 \pm 9.3$ & 0.21 \\
\hline BMI (kg/m2) & $23.70 \pm 2.99$ & $23.91 \pm 2.40$ & 0.63 \\
\hline Exposure to dust (former: never) & $22: 64$ & $20: 52$ & 0.84 \\
\hline Smoking index (pack-years) & $(0,22.5)$ & $(0,28.25)$ & 0.90 \\
\hline Clinical questionnaires & - & - & - \\
\hline mMRC & $0.17 \pm 0.41$ & $0.22 \pm 0.54$ & 0.53 \\
\hline CAT & $3.81 \pm 4.57$ & $4.36 \pm 4.74$ & 0.47 \\
\hline CCQ & $1.11 \pm 6.19$ & $1.31 \pm 6.27$ & 0.84 \\
\hline Spirometry & - & - & - \\
\hline FEV1 (L) & $2.71 \pm 0.67$ & $2.64 \pm 0.67$ & 0.47 \\
\hline FVC (L) & $3.31 \pm 0.78$ & $3.31 \pm 0.75$ & 0.93 \\
\hline FEV1/FVC & $0.82 \pm 0.07$ & $0.79 \pm 0.08$ & 0.01 \\
\hline FEV1\%prep & $0.92 \pm 0.14$ & $0.86 \pm 0.13$ & \\
\hline
\end{tabular}

Data are mean \pm standard deviation or median (IQR). p values comparing low-risk group and high-risk group are from two-sample t-test, Mann-Whitney $U$ test, $\chi^{2}$ or Fisher's exact test.

\section{Comparison Of Hrct Parameters}

A comparison of HRCT parameters between the two groups (low-risk group $n=86$, high-risk group $n=72$ ) is shown in Table 2. The mean values of LAA-950ins\% in the left lung, right lung, and the entire lung were $1.80 \pm 1.73,5.94 \pm 2.00$, and $5.92 \pm 1.98$ in the low-risk group, and $5.69 \pm 1.80,6.52 \pm 2.48$, and $6.36 \pm 1.70$ in the high-risk group, respectively. The value of LAA-950ins\%, called the emphysema index, showed a difference between two groups only in the left lung $(p=0.02)$, but there was no difference in the right lung 
or the entire lung $(p=0.11$ and $p=0.15)$. The mean LAA-856exp $\%$ in the left lung, right lung, and the entire lung were $14.75 \pm 5.90,14.36 \pm 6.14$, and $14.32 \pm 6.14$ in the low-risk group, and $19.61 \pm 6.59,21.54 \pm 6.78$, and $21.25 \pm 6.63$ in the high-risk group, respectively. The value of LAA-856exp\%, called the air trapping index, was significantly higher in the high-risk group than in the low-risk group $(p=0.00)$. The value of MLDins in the low-risk group was significantly higher than that in the high-risk group in the left lung $(-751.66 \pm 29.32$ vs. $-765.70 \pm 38.29, p=0.01)$ and in the entire lung $(-757.51 \pm 4.25$ vs. $-760.40 \pm 5.02, p=0.04)$. The value of MLDexp in the low-risk group was also significantly higher than that in the high-risk group in the left lung $(-732.76 \pm 44.08$ vs. $-745.26 \pm 35.24, p=0.04)$, in the right lung $(-738.13 \pm 36.89$ vs. $-754.69 \pm 30.50, p=0.00)$, and in the entire lung $(-735.86 \pm 39.10$ vs. $-750.49 \pm 31.91, p=0.01)$. 
Table 2

Comparison of HRCT parameters between the low-risk group and highrisk group

\begin{tabular}{|c|c|c|c|}
\hline & Low-risk group & High-risk group & $P$ value \\
\hline \multicolumn{4}{|c|}{ (ml) } \\
\hline Left lung & $77.92 \pm 36.10$ & $90.95 \pm 32.09$ & 0.02 \\
\hline Right lung & $90.97 \pm 39.57$ & $107.04 \pm 47.34$ & 0.02 \\
\hline Entire lung & $170.08 \pm 74.94$ & $195.97 \pm 70.93$ & 0.03 \\
\hline \multicolumn{4}{|l|}{ (ml) } \\
\hline Left lung & $1295.87 \pm 271.28$ & $1412.11 \pm 277.34$ & 0.02 \\
\hline Right lung & $1494.76 \pm 320.03$ & $1619.00 \pm 324.52$ & 0.02 \\
\hline Entire lung & $2806.53 \pm 600.09$ & $3041.12 \pm 590.16$ & 0.02 \\
\hline \multicolumn{4}{|c|}{$\%$} \\
\hline Left lung & $1.80 \pm 1.73$ & $5.69 \pm 1.80$ & 0.02 \\
\hline Right lung & $5.94 \pm 2.00$ & $6.52 \pm 2.48$ & 0.11 \\
\hline Entire lung & $5.92 \pm 1.98$ & $6.36 \pm 1.70$ & 0.15 \\
\hline \multicolumn{4}{|c|}{$(H U)$} \\
\hline Left lung & $-751.66 \pm 29.32$ & $-765.70 \pm 38.29$ & 0.01 \\
\hline Right lung & $-747.36 \pm 181.63$ & $-761.52 \pm 25.28$ & 0.48 \\
\hline Entire lung & $-757.51 \pm 4.25$ & $-760.40 \pm 5.02$ & 0.04 \\
\hline \multicolumn{4}{|c|}{ (ml) } \\
\hline Left lung & $162.34 \pm 118.40$ & $265.90 \pm 127.46$ & 0.00 \\
\hline Right lung & $(61.57,123.62)$ & $(140.49,371.61)$ & 0.00 \\
\hline Entire lung & $326.62 \pm 209.61$ & $582.05 \pm 270.14$ & 0.00 \\
\hline \multicolumn{4}{|c|}{ (ml) } \\
\hline Left lung & $997.28 \pm 266.62$ & $1191.13 \pm 275.22$ & 0.00 \\
\hline
\end{tabular}




\begin{tabular}{|llll|}
\hline & Low-risk group & High-risk group & P value \\
\hline Right lung & $1103.39 \pm 366.67$ & $1398.92 \pm 345.03$ & 0.00 \\
\hline Entire lung & $2104.40 \pm 561.51$ & $2612.62 \pm 606.93$ & 0.00 \\
\hline Left lung & & & \\
\hline Right lung & $14.75 \pm 5.90$ & $19.61 \pm 6.59$ & 0.00 \\
\hline Entire lung & $14.36 \pm 6.14$ & $21.54 \pm 6.78$ & 0.00 \\
\hline & & $21.25 \pm 6.63$ & 0.00 \\
\hline Left lung & $-732.76 \pm 44.08$ & $-745.26 \pm 35.24$ & 0.04 \\
\hline Right lung & $-738.13 \pm 36.89$ & $-754.69 \pm 30.50$ & 0.00 \\
\hline Entire lung & $-735.86 \pm 39.10$ & $-750.49 \pm 31.91$ & 0.01 \\
\hline
\end{tabular}

Data are mean \pm standard deviation or median (IQR). p values comparing low-risk group and high-risk group are from two-sample t-test, Mann-Whitney $U$ test, $\chi^{2}$ or Fisher's exact test.

\section{Correlations Between Quantitative Measurements And Pulmonary Function Parameters}

The correlations of various quantitative HRCT parameters at suspended full inspiration with the pulmonary function test variables are shown in Table 3. The LAA-950ins\% showed a strong correlation with the FEV1/FVC ( $r=-0.33$ in the left lung, $r=-0.22$ in the right lung, $r=-0.26$ in the entire lung, $p<0.05)$, a moderate correlation with FEV1 only in the left lung $(r=-0.19, p=0.04)$, and no correlation with $F V C$ or FEV1\%prep. TheLAA-950ins also showed a strong correlation with the FEV1/FVC ( $r=-0.33$ in the left lung, $r=-0.22$ in the right lung, $r=-0.26$ in the entire lung, $p<0.05)$, but no correlation with FEV1, FVC, or FEV1\%prep. TheLVins showed a statistical correlation with FVC ( $r=0.23$ in the left lung, $r=0.19$ in the right lung, $r=0.21$ in the entire lung, $p<0.05$ ), but no correlation with FEV1, FEV1/FVC, or FEV1\%prep. The MLDins in the left lung and in the entire lung showed a correlation with the FEV1/FVC $(r=0.20$ in the left lung and $r=0.21$ in the entire lung, $p<0.05$ ), but the MLDins in the right lung had no correlation with the FEV1/FVC $(p=0.06)$. There was no statistical correlation between the MLDins and three other physiologic parameters, including FEV1, FVC, and FEV1\%prep ( $p>0.05)$. 
Table 3

The correlation between quantitative HRCT parameters at inspiration and pulmonary function parameters

\begin{tabular}{|c|c|c|c|c|c|c|c|c|c|}
\hline & & & & FVC & & & vc & & prep \\
\hline & & $r$ & $P$ & $\mathrm{R}$ & $P$ & $r$ & $P$ & $r$ & $P$ \\
\hline \multirow[t]{4}{*}{ Left lung } & & -0.07 & 0.46 & 0.02 & 0.80 & $-0.33^{\star \star}$ & 0.00 & -0.12 & 0.19 \\
\hline & & 0.16 & 0.09 & $0.23^{\star}$ & 0.01 & $-0.23^{\star}$ & 0.01 & -0.07 & 0.47 \\
\hline & $\%$ & $-0.19 *$ & 0.04 & -0.11 & 0.26 & $-0.33^{\star \star}$ & 0.00 & -0.12 & 0.18 \\
\hline & & 0.07 & 0.48 & 0.01 & 0.91 & $0.20 *$ & 0.03 & 0.05 & 0.57 \\
\hline \multirow[t]{4}{*}{ Right lung } & & -0.02 & 0.81 & 0.05 & 0.60 & $-0.22^{\star}$ & 0.02 & -0.09 & 0.35 \\
\hline & & 0.15 & 0.12 & $0.19 *$ & 0.04 & -0.12 & 0.18 & -0.08 & 0.42 \\
\hline & $\%$ & -0.15 & 0.12 & -0.08 & 0.42 & $-0.22^{\star}$ & 0.02 & -0.09 & 0.35 \\
\hline & & 0.02 & 0.84 & -0.03 & 0.76 & 0.17 & 0.06 & 0.02 & 0.81 \\
\hline \multirow[t]{4}{*}{ Entire lung } & & 0.00 & 0.99 & 0.08 & 0.39 & $-0.26^{* *}$ & 0.01 & -0.09 & 0.34 \\
\hline & & 0.15 & 0.10 & $0.21^{*}$ & 0.02 & -0.18 & 0.05 & -0.08 & 0.40 \\
\hline & $\%$ & -0.12 & 0.20 & -0.05 & 0.63 & $-0.26^{\star \star}$ & 0.01 & -0.11 & 0.26 \\
\hline & & 0.07 & 0.45 & 0.01 & 0.94 & 0.21 * & 0.02 & 0.05 & 0.57 \\
\hline
\end{tabular}

** $p<0.01, * p<0.05$

The LAA-856exp\% showed a significantly strong correlation with FEV1, FEV1/FVC, and FEV1\%prep $(p<0.05)$ in Table 4. Among them, the most optimal correlation between LAA-856exp\% was found with FEV1/FVC ( $r=-0.33$ in the left lung, $r=-0.23$ in the right lung, and $r=-0.28$ in the entire lung). No useful correlation was found between the LAA-856exp\% and FVC. Both LAA-856exp and LVexp showed statistical correlation with FEV1/FVC ( $r=-0.32$ in the left lung, $r=-0.23$ in the right lung, $r=-0.28$ in the entire 
lung, and $r=-0.29$ in the left lung, $r=-0.17$ in the right lung, $r=-0.25$ in the entire lung, respectively, $p<0.05$ ). The LAA-856exp\% and LVexp also showed correlation with FEV1\%prep ( $r=-0.24$ in the left lung, $r=-0.26$ in the right lung, $r=-0.25$ in the entire lung and $r=-0.25$ in the left lung, $r=-0.24$ in the right lung, $r=-0.27$ in the entire lung, respectively, $p<0.05$ ), but neither of them correlated with FEV1or FVC. For the MLDexp, strong and positive correlations were found with FEV1/FVC ( $r=0.31$ in the left lung, $r=0.25$ in the right lung and $r=0.29$ in the entire lung, $p<0.01$ ), while no statistical correlation was found with FEV1, FVC, and FEV1\%prep ( $p>0.05)$. 
Table 4

The correlation between quantitative HRCT parameters at expiration and pulmonary function parameters

\begin{tabular}{|c|c|c|c|c|c|c|c|c|}
\hline & & & FVC & & & VC & & prep \\
\hline & $r$ & $P$ & $\mathrm{R}$ & $P$ & $r$ & $P$ & $r$ & $P$ \\
\hline \multirow{4}{*}{$\begin{array}{l}\text { Left } \\
\text { lung }\end{array}$} & -0.13 & 0.14 & -0.09 & 0.34 & $-0.32 \star \star$ & 0.00 & $-0.24^{\star \star}$ & 0.01 \\
\hline & -0.06 & 0.52 & -0.01 & 0.92 & $-0.29 * \star$ & 0.01 & $-0.25^{\star \star}$ & 0.01 \\
\hline & $-0.19 *$ & 0.04 & -0.15 & 0.11 & $-0.33^{\star *}$ & 0.00 & $-0.24^{\star \star}$ & 0.01 \\
\hline & -0.06 & 0.50 & -0.14 & 0.13 & 0.31 ** & 0.00 & 0.03 & 0.73 \\
\hline \multirow{4}{*}{$\begin{array}{l}\text { Right } \\
\text { lung }\end{array}$} & -0.16 & 0.08 & -0.13 & 0.16 & $-0.23^{\star}$ & 0.01 & -0.26 ** & 0.00 \\
\hline & -0.08 & 0.38 & -0.06 & 0.50 & $-0.17 *$ & 0.03 & $-0.24^{\star}$ & 0.01 \\
\hline & -0.20 * & 0.03 & -0.17 & 0.08 & $-0.23^{\star}$ & 0.01 & $-0.25^{\star \star}$ & 0.01 \\
\hline & -0.06 & 0.49 & -0.13 & 0.15 & $0.25^{\star \star}$ & 0.00 & 0.04 & 0.69 \\
\hline \multirow{4}{*}{$\begin{array}{l}\text { Entire } \\
\text { lung }\end{array}$} & -0.14 & 0.14 & -0.10 & 0.29 & $-0.28^{\star \star}$ & 0.00 & $-0.25^{\star \star}$ & 0.01 \\
\hline & -0.09 & 0.34 & -0.05 & 0.59 & $-0.25^{\star \star}$ & 0.01 & $-0.27 * \star$ & 0.00 \\
\hline & -0.20 * & 0.03 & -0.16 & 0.08 & $-0.28 * \star$ & 0.00 & $-0.24^{\star \star}$ & 0.01 \\
\hline & -0.08 & 0.41 & -0.15 & 0.10 & $0.29 \star \star$ & 0.00 & 0.02 & 0.80 \\
\hline
\end{tabular}

** $p<0.01, * p<0.05$

\section{Discussion}

Our observational study on 158 patients who were at risk of developing COPD demonstrates that HRCT measurements of emphysema index and air trapping index were negatively correlated with FEV1/FVC from PFT, whereas the mean expiratory lung density showed a positive correlation. Patients in the high- 
risk group exhibited a significantly higher air trapping index but lower mean expiratory lung density than those in the low-risk group.

COPD is a chronic inflammatory airway disease that is characterized by airflow limitations that are not fully reversible [17]. At the early stage of the disease, patients are often asymptomatic or exhibit only mild chronic cough or dyspnea $[18,19]$. However, as the disease progresses, patients may experience chest pain, expectoration, fatigue, weight loss, and can also develop acute lower respiratory infections, cardiovascular disease, or lung cancer that continue to place an enormous burden on society [20, 21]. According to the latest research, the prevalence of COPD in China is continually increasing, and the subgroup of patients over 40 years of age has nearly 99 million people, accounting for $13.7 \%$ [3]. Therefore, early detection and timely management are imperative.

There has been interest in the diagnostic value of HRCT for COPD, and its combined use with advanced postprocessing software will provide important clinical applications for COPD. Several studies [22-24] have established the relationship between lung function indicators such as FEV1/FVC, RV/TLC, and CT findings. Some CT scans have been previously used to classify the severity in COPD patients. However, articles discussing CT findings in patients at risk of developing COPD are scarce. The present study shows that the HRCT emphysema index, air trapping index, and the mean expiratory lung density each contain independent diagnostic information for patients at risk of developing COPD, and represent independent imaging biomarkers $[25,26]$.

We find from Table 1 that compared with the low-risk group, there was no statistically significant difference in the clinical features (age, sex, BMI categories, dust exposure, and smoking index) and the clinical symptom scores (mMRC, CAT, and CCQ) for the high-risk group. However, the differences in the forced expiratory volume in the first second/forced vital capacity and forced expiratory volume in the first second as a percentage of the expected value showed significant difference $(P<0.01)$ between the two groups. This suggests that based on behavioral observation and recordings, the severity of COPD in the different states was indistinguishable. These results were in agreement with the findings of Sunmin Kim et al [27]. Their conclusion shows that the choice of symptom scale can alter the group assignment of COPD. The clinical features and the clinical symptom scores can be used to assist in gauging the condition, but there were limitations to the evaluation at the individual level. It is clear that additional indicators need to be comprehensively assessed in combination.

Comparing the CT parameters of the low-risk and high-risk COPD groups, the CT images of patients from the high-risk group exhibit certain clinical characteristics and typical imaging features. Higher lung volume and emphysema index, and lower mean lung density in the inspiratory phase and expiratory phase were present. This result demonstrates that in COPD high-risk patients, pulmonary hyperinflation and lung volume increased. Patients in the high-risk group have a significantly higher air trapping index but lower mean expiratory lung density as compared to the low-risk group. As for the emphysema index and the mean inspiratory lung density, this trend in the high and low-risk group was not statistically significant. Therefore, it is suggested that the air trapping index was more accurate at predicting the risk 
state of COPD and lung function decline than the emphysema index. In other words, using HRCT imaging for the study of lung pathologies in the early stage of the disease, the CT scans obtained at expiration were more informative than those at inspiration. In previous studies, the choice of an end inspiration or end expiration image remained controversial [28]. Some demonstrated the greater diagnostic sensitivity of inspiratory lung volumes for COPD [29]. CT measurements of airway dimensions and emphysema are useful and complementary in the evaluation of the lung with COPD.

Our study on the correlation between CT parameters and lung function shows that the left, right, and bilateral lung emphysema index and air trapping index were significantly inversely correlated with FEV1/FVC. This indicates that the emphysema index theoretically has the potential to use HRCT to assess evidence of emphysema and small airway disease. Our results indicate that the presence of increasing lung volume, persistent airflow limitation, and air trapping can also be present in those with undiagnosed COPD, which can in turn lead to lung function changes. In our study, we also found that the left, right, and bilateral lung mean expiratory lung density showed significantly positive correlation with FEV1/FVC. This is in accordance with the conclusion arrived at by previously published studies [30-32]. There was high predictive value of CT density parameters for detecting pulmonary ventilation.

Our study has several limitations. First, the study was conducted in a single center in China and was carried out at an outpatient clinic where patients had morbid conditions, and thus, inpatients were not included in the study. Second, the study population was relatively small and predominantly male. The results might be different in a larger population. Third, we did not enroll patients with confirmed COPD as controls, and therefore, the extrapolation of these findings to COPD cohorts must be performed with care. Last, no follow-up of the patients was performed.

\section{Conclusions}

In summary, our original study demonstrates that the combination of the air trapping index and the mean expiratory lung density obtained by HRCT can be used as an indicator for distinguishing between high and low risk of developing COPD. Emphysema index, air trapping index, and the mean expiratory lung density exhibited significant correlation with FEV1/FVC, which can be used to assess the pulmonary function status of people at risk of developing COPD, and provide a useful supplement for the early and comprehensive assessment of the disease.

\section{Abbreviations}

COPD

chronic obstructive pulmonary disease

CT

computed tomography

HRCT

high-resolution computed tomography 
$\mathrm{HU}$

housfield unites

PFTs

pulmonary function tests

FVC

forced vital capacity

FEV1

forced expiratory volume in first second

FEV1\%prep

forced expiratory volume in first second as a percentage of the expected value FEV1/FVC

forced expiratory volume in first second/forced vital capacity

LAA

low attenuation areas

LAA-856exp

low attenuation areas less than-856HU on expiratory CT scan

LAA-950ins

low attenuation areas less than-950HU on inspiratory CT scan

LAA-856exp\%

low attenuation areas less than-856HU to lung volume on expiratory CT scan

LAA-950ins\%

low attenuation areas less than-950HU to lung volume on inspiratory CT scan

MLD

mean lung density

MLD $\exp$

mean lung density on expiratory CT scan

$M L D_{\text {ins }}$

mean lung density on inspiratory CT scan

$\mathrm{LV}_{\text {ins }}$

lung volume on inspiratory CT scan

$\mathrm{LV}_{\text {exp }}$

lung volume on expiratory CT scan

mMRC

modified British medical research council

CAT

COPD assessment test

CCQ

clinical COPD questionnaire

El 
emphysema index

ATI

air trapping index

RV/TLC

residual volume/total lung capacity.

\section{Declarations}

\section{Ethics approval and consent to participate}

This retrospective and single-center cohort study was carried out at The First Affiliated Hospital of Wenzhou Medical University in China. Ethical approval was obtained, and all participants consented to be included in the trial.

\section{Consent for publication}

All list authors consent to the submission and all data are used with the consent of the person generating the data.

\section{Competing interests}

The authors declare that they have no competing interests.

\section{Authors' contributions}

LY, XH and LW conceived and designed the study, responsible for the integrity and accuracy of the data, and had full access to the study. RL and $\mathrm{HJ}$ contributed to drafting and writing this paper. MX took responsibility for obtaining written consent from patients, obtaining ethical approval, collecting samples, and confirming data accuracy. PS, YW and DY made substantial contributions to data acquisition, analysis, and interpretation. All the authors had strictly revised the manuscript and agreed to be responsible for all aspects of the work, and finally approved the version to be published.

\section{Acknowledgements}

Not applicable.

\section{References}

1. Coxson HO, Dirksen A, Edwards LD, Yates JC, Agusti A, Bakke P, Calverley PM, Celli B, Crim C, Duvoix $A$, et al: The presence and progression of emphysema in COPD as determined by CT scanning and 
biomarker expression: a prospective analysis from the ECLIPSE study. Lancet Respir Med 2013, 1:129-136.

2. López-Campos JL, Tan W, Soriano JB: Global burden of COPD. Respirology 2016, 21:14-23.

3. Fang L, Gao P, Bao H, Tang X, Wang B, Feng Y, Cong S, Juan J, Fan J, Lu K, et al: Chronic obstructive pulmonary disease in China: a nationwide prevalence study. Lancet Respir Med 2018, 6:421-430.

4. Duffy SP, Criner GJ: Chronic Obstructive Pulmonary Disease: Evaluation and Management. Med Clin North Am 2019, 103:453-461.

5. Pratt PC, Kilburn KH: A modern concept of the emphysemas based on correlations of structure and function. Hum Pathol 1970, 1:443-463.

6. Regan EA, Lynch DA, Curran-Everett D, Curtis JL, Austin JH, Grenier PA, Kauczor HU, Bailey WC, DeMeo DL, Casaburi RH, et al: Clinical and Radiologic Disease in Smokers With Normal Spirometry. JAMA Intern Med 2015, 175:1539-1549.

7. Singh D, Agusti A, Anzueto A, Barnes PJ, Bourbeau J, Celli BR, Criner GJ, Frith P, Halpin DMG, Han M, et al: Global Strategy for the Diagnosis, Management, and Prevention of Chronic Obstructive Lung Disease: the GOLD science committee report 2019. Eur Respir J 2019, 53.

8. Labaki WW, Han MK: Improving Detection of Early Chronic Obstructive Pulmonary Disease. Ann Am Thorac Soc 2018, 15:S243-s248.

9. Kalra MK, Ebrahimian S: Quantitative Chest CT in COPD: Can Deep Learning Enable the Transition? Radiol Cardiothorac Imaging 2021, 3:e210044.

10. Xu Y, Yamashiro T, Moriya H, Muramatsu S, Murayama S: Quantitative Emphysema Measurement On Ultra-High-Resolution CT Scans. Int J Chron Obstruct Pulmon Dis 2019, 14:2283-2290.

11. Madani A, Zanen J, de Maertelaer V, Gevenois PA: Pulmonary emphysema: objective quantification at multi-detector row CT--comparison with macroscopic and microscopic morphometry. Radiology 2006, 238:1036-1043.

12. Nambu A, Zach J, Schroeder J, Jin G, Kim SS, Kim YI, Schnell C, Bowler R, Lynch DA: Quantitative computed tomography measurements to evaluate airway disease in chronic obstructive pulmonary disease: Relationship to physiological measurements, clinical index and visual assessment of airway disease. Eur J Radiol 2016, 85:2144-2151.

13. Matsuoka S, Kurihara Y, Yagihashi K, Hoshino M, Watanabe N, Nakajima Y: Quantitative assessment of air trapping in chronic obstructive pulmonary disease using inspiratory and expiratory volumetric MDCT. AJR Am J Roentgenol 2008, 190:762-769.

14. Bhatt SP, Soler X, Wang X, Murray S, Anzueto AR, Beaty TH, Boriek AM, Casaburi R, Criner GJ, Diaz AA, et al: Association between Functional Small Airway Disease and FEV1 Decline in Chronic Obstructive Pulmonary Disease. Am J Respir Crit Care Med 2016, 194:178-184.

15. Lynch DA: Progress in Imaging COPD, 2004 - 2014. Chronic Obstr Pulm Dis 2014, 1:73-82.

16. Bhaskar R, Singh S, Singh P: Characteristics of COPD phenotypes classified according to the findings of HRCT and spirometric indices and its correlation to clinical characteristics. Afr Health Sci 2018, 
18:90-101.

17. Rabe KF, Watz H: Chronic obstructive pulmonary disease. Lancet 2017, 389:1931-1940.

18. Casaburi R, Duvall K: Improving early-stage diagnosis and management of COPD in primary care. Postgrad Med 2014, 126:141-154.

19. Rokach A, Bohadana A, Kotek O, Shuali CC, Azulai H, Babai P, Freier-Dror Y, Izbicki G: Early Detection of COPD: An Opportunistic Case Finding Study in Smokers and Ex-Smokers Visiting a Medical Centre. Int J Chron Obstruct Pulmon Dis 2021, 16:1519-1527.

20. Hurst JR, Siddiqui MK, Singh B, Varghese P, Holmgren U, de Nigris E: A Systematic Literature Review of the Humanistic Burden of COPD. Int J Chron Obstruct Pulmon Dis 2021, 16:1303-1314.

21. Løkke A, Lange P, Lykkegaard J, Ibsen R, Andersson M, de Fine Licht S, Hilberg O: Economic Burden of COPD by Disease Severity - A Nationwide Cohort Study in Denmark. Int J Chron Obstruct Pulmon Dis 2021, 16:603-613.

22. Koo HJ, Lee SM, Seo JB, Lee SM, Kim N, Oh SY, Lee JS, Oh YM: Prediction of Pulmonary Function in Patients with Chronic Obstructive Pulmonary Disease: Correlation with Quantitative CT Parameters. Korean J Radiol 2019, 20:683-692.

23. Paoletti M, Cestelli L, Bigazzi F, Camiciottoli G, Pistolesi M: Chronic Obstructive Pulmonary Disease: Pulmonary Function and CT Lung Attenuation Do Not Show Linear Correlation. Radiology 2015, 276:571-578.

24. Genseke P, Wetz C, Wallbaum T, KreissI MC, Ghazzawi S, Schreiber J, Amthauer H, Grosser OS: Preoperative quantification of pulmonary function using hybrid-SPECT/low-dose-CT: A pilot study. Lung Cancer 2018, 118:155-160.

25. Lee YK, Oh YM, Lee JH, Kim EK, Lee JH, Kim N, Seo JB, Lee SD: Quantitative assessment of emphysema, air trapping, and airway thickening on computed tomography. Lung 2008, 186:157-165.

26. Pompe E, Strand M, van Rikxoort EM, Hoffman EA, Barr RG, Charbonnier JP, Humphries S, Han MK, Hokanson JE, Make BJ, et al: Five-year Progression of Emphysema and Air Trapping at CT in Smokers with and Those without Chronic Obstructive Pulmonary Disease: Results from the COPDGene Study. Radiology 2020, 295:218-226.

27. Kim S, Oh J, Kim YI, Ban HJ, Kwon YS, Oh IJ, Kim KS, Kim YC, Lim SC: Differences in classification of COPD group using COPD assessment test (CAT) or modified Medical Research Council (mMRC) dyspnea scores: a cross-sectional analyses. BMC Pulm Med 2013, 13:35.

28. Zaporozhan J, Ley S, Eberhardt R, Weinheimer O, lliyushenko S, Herth F, Kauczor HU: Paired inspiratory/expiratory volumetric thin-slice CT scan for emphysema analysis: comparison of different quantitative evaluations and pulmonary function test. Chest 2005, 128:3212-3220.

29. Zhang D, Guan Y, Fan L, Xia Y, Liu SY: [Quantitative analysis of emphysema and air trapping at inspiratory and expiratory phase multi-slice spiral CT scan in smokers: correlation with pulmonary function test]. Zhonghua Yi Xue Za Zhi 2018, 98:1467-1473.

30. Shaker SB, Dirksen A, Ulrik CS, Hestad M, Stavngaard T, Laursen LC, Maltbaek N, Clementsen P, Skjaerbaek N, Nielsen L, et al: The effect of inhaled corticosteroids on the development of 
emphysema in smokers assessed by annual computed tomography. Copd 2009, 6:104-111.

31. Zhang L, Wang Y: [Sixty-four slice spiral CT low-dose chest two-phase scanning for lung density measurement in assessing the pulmonary function in patients with chronic obstructive pulmonary disease]. Zhong Nan Da Xue Xue Bao Yi Xue Ban 2012, 37:1156-1162.

32. Cheng T, Li Y, Pang S, Wan H, Shi G, Cheng Q, Li Q, Pan Z, Huang S: Normal lung attenuation distribution and lung volume on computed tomography in a Chinese population. Int $\mathrm{J}$ Chron Obstruct Pulmon Dis 2019, 14:1657-1668.

\section{Figures}




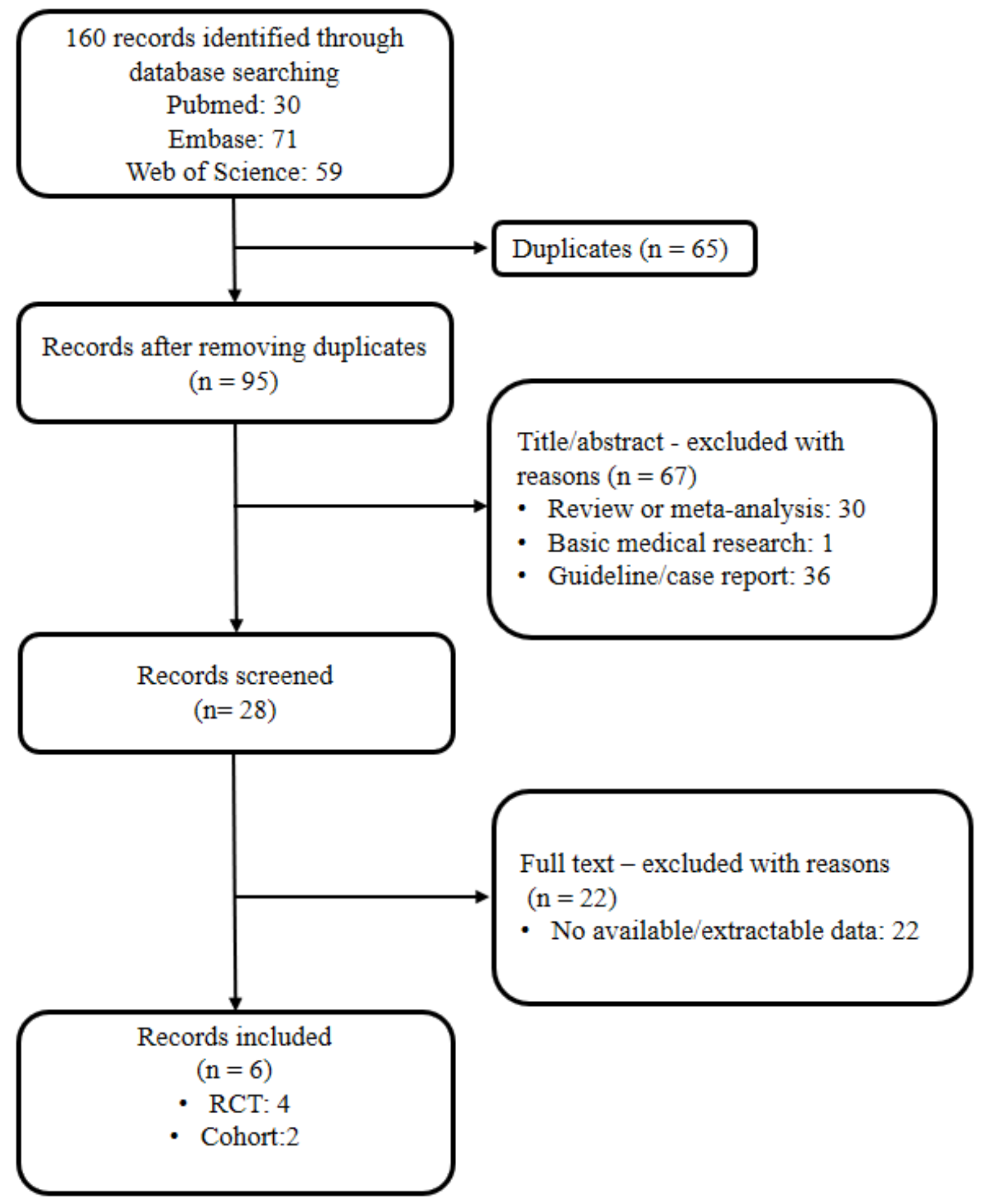

Figure 1

Study flow diagram 